\title{
The emerging role of the genomics revolution in agricultural biotechnology
}

\section{Jonathan Margolis and Geoffrey Duyk}

Although those involved in the therapeutics and agricultural businesses rarely acknowledge the similarities of their enterprises, the impact of genomics is forcing this realization. In our view, the agrichemical industry is on the verge of shifting from chemistry-based, whole organism screening methods, which it-like the pharmaceutical industry - has used for over 50 years, to biologically driven, targetbased screening methods currently revitalizing the search for new therapeutics. The convergence of classical biology, genomics tools, and sequence information, and the availability of sophisticated model genetic systems, offer agricultural biotechnology a unique opportunity to redefine the way in which pesticides are discovered. The cross-fertilization between genomics research in agricultural biotechnology and therapeutic biotechnology merits close attention as both these industries find their way in the brave new world of genomics.

\section{Parallel paths}

Many parallels can be drawn between the two industries in their approaches to research and development. Until recently, agricultural companies, much like their pharmaceutical brethren, have relied on either modification of the chemistry of existing compounds, or direct screening of chemical entities against whole organisms to fill their pipeline with new compounds. Over the past 50 years, these two processes have resulted in a relatively small number of new classes of pesticide and a gradual modification of the chemistry of existing compounds to produce incrementally better pesticides. Thus, as of 1995 , three-quarters of all pesticide sales worldwide were accounted for by compounds acting on just two targets: acetylcholinesterase and the sodium channel'. This reliance on incremental improvement in the research and development path was similar to the so-called "me too" approach to drug development in the pharmaceutical industry: Drug developers relied on profits from therapeutics that offered marginal improvements over a competitor's product.

The current challenge facing the pesticide

\footnotetext{
Jonathan Margolis is agricultural biotechnology program leader, and Geoffrey Duyk is chief scientific officer at Exelixis Pharmaceuticals, Inc., South San Francisco,CA (duyk@exelixis.com).
}

industry is analogous to that confronted by the pharmaceutical industry in its quest for novel antibiotics. In both cases, the goal is to selectively kill or disable an invading organism with small-molecule toxins or drugs. Antibiotic research is also driven by the development of resistant strains and the need for drugs with new modes of action. Despite extensive screening over the last 50 years for novel compounds that kill or inhibit bacterial growth, the majority of antibiotics are directed against protein synthesis or cell-wall biosynthesis.

Although subject to very different market and regulatory pressures, both industries discovered that these traditional methods of finding new products were not sufficient to maintain their pipelines ${ }^{2}$. The most promising alternative is to identify and validate biological targets against which potential ligands can be screened, and in fact the pharmaceutical industry has widely adopted target-based screening as its primary discovery engine. In our view, a similar shift is inevitable in the agrichemical industry.

\section{Probing pathogen genetics}

Pesticide development has focused on the chemical and physical attributes of the pesticide itself. Target-based screening emphasizes the careful selection and validation of genes based on their suitability as pesticide targets. The ideal target is essential for viability and should be required throughout the life cycle. Inhibition or hyperactivation of the target should result in rapid death or paralysis of the pest (knockdown) as this halts crop destruction immediately upon treatment and is highly valued by farmers. The target should also be highly dose sensitive so that only a partial interference with its function causes knockdown. Highly divergent genes provide greater opportunities for chemical specificity that can minimize toxicity to other organisms such as vertebrates or plants. Finally, the target must be a member of a protein family amenable to formatting in a high-throughput biochemical or cell-based assay.

The availability of a complete genomic sequence in an increasing number of genetically manipulatable organisms will allow the testing of all genes as possible targets in these species. For example, work is already under way to systematically knock out and test the function of every gene in the yeast $S$. cerevisiae, and similar projects are contemplated in $C$. elegans and several bacterial species. Even in species such as Drosophila, where systematic knockout of every gene is impractical, targets can be identified and validated by classical genetic screens, existing mutant collections, or focused knockout screens. Current genomics and genetics technologies offer a clear pathway for establishing novel model systems based on insect or nematode species more closely related to pest species, as well as the possibility of developing germline transformation systems and/or viral gene transfer systems adapted for these next-generation model systems. This research will lead to the ability to define an optimal set of targets and then to develop assays that screen for inhibitors or agonists.

\section{Mutual benefit}

Exelixis is devoted to elucidating the genetic pathways important in the development and progression of human disease by using the tools of invertebrate genetics and genomics. These research programs offer a unique source of targets for pest control in pathways that would never be explored by traditional invertebrate biochemists. For example, in our neurodegenerative disease program, where we study Alzheimer's disease, only genes identified as having a loss of function phenotype that enhances neuronal survival would be candidates for developing human therapeutics. However, genes that result in neuronal death or dysfunction when knocked out provide potential pesticide targets. Because we are studying parallel pathways in vertebrates and invertebrates, we can focus our search for pesticide targets on the points at which these pathways differ. Many of our current research programs (e.g., insulin signaling or cell-cycle control) represent novel avenues of research in insect biology likely to result in the identification of new targets for pesticide development.

\section{Riding the wave}

For all their differences, the pharmaceutical and agrichemical industries depend on a continuous source of new lead compounds. The discovery of new therapeutics has been revolutionized over the last decade by the conjunction of genomics, high-throughput screening, and combinatorial chemistry. Like it or not, the agrichemical industry is going to be swept by the same wave.

\footnotetext{
1. Hopkins, W.L. 1996. Global Insecticide Directory. Ag Chem Info. Services. Indianapolis, IN.

2. Drews, J. 1996. Nature Biotechnology. 11:1516.
} 